\title{
BMJ
}

\section{Inpatient rehabilitation specifically designed for geriatric patients: systematic review and meta-analysis of randomised controlled trials}

\begin{abstract}
Stefan Bachmann, Robert Bosch Foundation postdoctoral research fellow in geriatrics, ${ }^{1,2}$ Christoph Finger, doctoral student, ${ }^{1}$ Anke Huss, assistant professor in environmental epidemiology, ${ }^{3,4}$ Matthias Egger, professor of epidemiology and public health, ${ }^{3,5}$ Andreas EStuck, professor of geriatrics, ${ }^{1}$ Kerri M Clough-Gorr, senior research fellow in epidemiology and geriatrics ${ }^{1,3,6}$
\end{abstract}

${ }^{1}$ Department of Geriatrics, Inselspital, University of Bern Hospital, Freiburgstrasse 10, CH-3010 Bern, Switzerland

${ }^{2}$ Rehabilitation Centre Klinik Valens, CH-7317 Valens,

Switzerland

${ }^{3}$ Institute of Social and Preventive Medicine, University of Bern, Finkenhubelweg 11, CH-3012 Bern, Switzerland

${ }^{4}$ Institute for Risk Assessment Sciences, Netherlands Utrecht University, PO Box 80178,

3508 TD, Utrecht, Netherlands

${ }^{5}$ Department of Social Medicine, University of Bristol, Bristol BS8 2PR

${ }^{6}$ Section of Geriatrics, Boston University Medical Center, Boston, MA 02118, USA

Correspondence to: A Stuck andreas.stuck@spitalnetzbern.ch

Cite this as: $B M J$ 2010;340:c1718 doi:10.1136/bmj.c1718

\section{ABSTRACT}

Objective To assess the effects of inpatient rehabilitation specifically designed for geriatric patients compared with usual care on functional status, admissions to nursing homes, and mortality.

Design Systematic review and meta-analysis.

Data sources Medline, Embase, Cochrane database, and reference lists from published literature.

Review methods Only randomised controlled trials were included. Trials had to report on inpatient rehabilitation and report at least one of functional improvement, admission to nursing homes, or mortality. Trials of consultation or outpatient services, trials including patients aged $<55$, trials of non-multidisciplinary rehabilitation, and trials without a control group receiving usual care were excluded. Data were double extracted. Odds ratios and relative risks with $95 \%$ confidence intervals were calculated.

Results 17 trials with 4780 people comparing the effects of general or orthopaedic geriatric rehabilitation programmes with usual care were included. Metaanalyses of effects indicated an overall benefit in outcomes at discharge (odds ratio 1.75 (95\% confidence interval 1.31 to 2.35 ) for function, relative risk 0.64 (0.51 to 0.81 ) for nursing home admission, relative risk 0.72 (0.55 to 0.95$)$ for mortality) and at end of follow-up (1.36 (1.07 to 1.71), 0.84 (0.72 to 0.99 ), 0.87 (0.77 to 0.97), respectively). Limited data were available on impact on health care or cost. Compared with those in control groups, weighted mean length of hospital stay after randomisation was longer in patients allocated to general geriatric rehabilitation ( $24.5 \vee 15.1$ days) and shorter in patients allocated to orthopaedic rehabilitation ( $24.6 \mathrm{~V}$ 28.9 days).

Conclusion Inpatient rehabilitation specifically designed for geriatric patients has the potential to improve outcomes related to function, admission to nursing homes, and mortality. Insufficient data are available for defining characteristics and cost effectiveness of successful programmes.

\section{INTRODUCTION}

As a complement to acute and curative medicine, rehabilitation medicine is recognised as an efficient tool in the treatment of patients in the Western world. According the World Health Organization, the aim of rehabilitation is to maximise function and minimise limitation of activity and restriction of participation resulting from an underlying impairment or disease. ${ }^{1}$ WHO's international classification of functioning, disability, and health (ICF) framework takes into account not just medical or biological dysfunction but the social aspects of disability as well. It shifts the focus of rehabilitation medicine from cause to impact and places all health conditions on an equal footing in assessing the impact of multiple domains on a person's functioning.

Rehabilitation medicine is well accepted as a specialised medical discipline for the treatment of younger patients with organ specific neurological, musculoskeletal, orthopaedic, pulmonary, and cardiovascular diseases. In geriatric patients with special needs associated with ageing (such as cognitive problems, multiple comorbidities, polypharmacy, end of life decisions) the situation is less clear, and the impact of rehabilitation (for instance, effect on health outcomes, rates of readmission to hospital, healthcare cost-benefit) is still controversial. Although it is well known that after an acute hospital stay older adults are at increased risk of death and admission to a nursing home ${ }^{23}$ inpatient rehabilitation specifically designed for older adults to address the special aspects of ageing is not standard practice.

Specialised inpatient and outpatient treatment for older adults might have the potential to optimise health outcomes, notably by improving functional status. As a result, geriatric rehabilitation programmes might not only improve outcomes but might also generate long term cost savings by reducing admissions to nursing homes. ${ }^{45}$ Furthermore, delaying functional decline and avoiding such admissions might be instrumental not just in reducing healthcare costs but also in effectively maintaining quality of life in older adults. ${ }^{6}$ 
To the best of our knowledge there are no systematic reviews or meta-analyses focusing on the topic of inpatient rehabilitation of older adults based on the WHO framework. We summarised the short term (at discharge) and longer term (at end of follow-up) effects of inpatient rehabilitation specifically designed for geriatric patients on the key outcomes of functional improvement, admissions to nursing homes, and mortality. We also identified characteristics that might differentiate successful from unsuccessful rehabilitation programmes as well as their impact on health care. We hypothesised that inpatient rehabilitation programmes are more likely to be beneficial if they follow the WHO framework rehabilitation cycle. ${ }^{7-9}$ Such programmes include a multidimensional geriatric assessment, stringent assignment to therapies, regular team meetings with all health professionals involved in the care of the patient, goal setting tailored to the individual patient, interventions tailored to the patient's needs, and regular treatment evaluation with the care team and the patient.

\section{METHODS}

\section{Literature search and eligibility criteria}

We searched for randomised controlled trials on the effects of inpatient rehabilitation specifically designed for geriatric patients. Rehabilitation was defined as inpatient multidisciplinary programmes with active physiotherapy or occupational therapy, or both, according the WHO ICF framework. Published studies were identified through searches in Medline, Embase (1 January 1970 to 31 July 2008), and the Cochrane Central Register of Controlled Trials (CENTRAL) database using the key words: geriatric, elderly, older, old, very old, aged, inpatient, rehabilitation, discharge, post(-) acute, hospitaliz(s)ed, randomiz(s)ed in combination with falls prevention, exercise, fitness, training, multidisciplinary therapy, ICF, physiotherapy, occupational therapy, geriatric evaluation, geriatric assessment, geriatric management, nursing home, mortality, Barthel, Functional Independence Measure (FIM), Mini mental, Timedup-and-go-test, function, functional, fit-to-walk, restoration, strength, maintenance.

Additional trials were identified by screening reference lists. No language restrictions were applied. Trials had to report on inpatient rehabilitation specifically designed for geriatric patients and report at least one of functional status, admissions to nursing homes, or mortality. We excluded studies that were not peer reviewed randomised controlled trials, studies offered to patients of all ages (that is, the study did not use an age threshold for including patients or the study used an age threshold of $<55$ ), interventions that were not an inpatient programme in a designated unit, interventions that were part of an acute care programme without rehabilitation in medically stable patients, interventions that did not include a multidisciplinary therapy programme including active physiotherapy or occupational therapy, or both, directed towards functional status of patients, studies that did not report on the predefined outcome data, and studies with a control group in which patients did not receive usual care (such as randomised controlled trials comparing two different forms of rehabilitation).

\section{Data extraction and management}

Two reviewers (SB and $\mathrm{CF}$ ) independently screened titles, abstracts, and full texts. Discrepancies were resolved through discussion with a third reviewer (AES). We extracted information on the mean age of the study population, location of the intervention (such as separate ward in acute hospital, separate hospital for rehabilitation), mean length of stay in hospital under acute care before randomisation (for intervention and control groups combined), length of hospital stay after randomisation (separately for intervention and control group), length of follow-up for outcome evaluation, and whether or not patients in the intervention group entered an outpatient follow-up therapy programme after their stay in hospital. Most trials reported length of hospital stay after randomisation as a mean or median value without standard deviations. We therefore calculated means within groups weighted for sample size; statistical pooling of the data was not possible.

On the basis of search results, we classified studies as orthopaedic geriatric rehabilitation after hip fracture or general geriatric rehabilitation. We extracted all information on the key outcomes of functional status, admissions to nursing homes, and mortality at discharge (or within four weeks after discharge) and at the end of follow-up (preferably at one year or closest to one year). Our definition of functional status was based on activities of daily living (ADL), most commonly the Barthel index ${ }^{10}$ (six trials), ${ }^{11-19}$ or the Katz index ${ }^{20}$ (six trials), ${ }^{21-27}$ but also included other measures of functional ability as reported (for example, activities of daily living score, personal self maintenance scale). ${ }^{528-30}$ If more than one functional outcome was reported we used measures based on activities of daily living score. At discharge and at the end of follow-up we recorded the number of patients with functional improvement, the number admitted to nursing homes, and the number who died. Unpublished data were available from three studies. ${ }^{212829}$ Lastly, we measured the impact of geriatric rehabilitation programmes on health care measured by rate of readmission to hospital and total programme costs.

\section{Assessment of quality of intervention programme and methodological quality of trials}

Two reviewers (SB and $\mathrm{CF}$ ) independently assessed all included trials for quality of the intervention programme and the trial methods. Quality of the intervention programme was measured according to the WHO ICF framework rehabilitation cycle. It included whether or not studies performed multidimensional geriatric assessment and assigned patients (defined as assignment to therapy and care team meetings for individualised goal setting). Assessment of methodological quality was based on concealment of allocation (for instance, explicit report of a method of concealed random allocation), independence of assessors (for instance, explicit report 
Table 1|Description of main characteristics of 17 included randomised controlled trials (listed alphabetically by type of rehabilitation) of inpatient rehabilitation specifically designed for geriatric patients. In all studies, patients in control group received standard inpatient care, except in study of Karppi et al, ${ }^{22}$ in which they received usual supervised home care

\begin{tabular}{|c|c|c|c|c|c|c|}
\hline & $\begin{array}{c}\text { Type of } \\
\text { hospital for } \\
\text { initial acute } \\
\text { care }\end{array}$ & $\begin{array}{l}\text { Reason for initial } \\
\text { hospital } \\
\text { admission }\end{array}$ & $\begin{array}{c}\text { Mean length of } \\
\text { hospital stay before } \\
\text { randomisation (days) }\end{array}$ & Main criteria for selection of study sample & $\begin{array}{l}\text { Mean age } \\
\text { (years) }\end{array}$ & $\begin{array}{l}\text { No of patients } \\
\text { (intervention/ } \\
\text { control) }\end{array}$ \\
\hline \multicolumn{7}{|c|}{ General geriatric rehabilitation } \\
\hline $\begin{array}{l}\text { Applegate 1990, } \\
\text { USA }^{2843}\end{array}$ & $\begin{array}{l}\text { Community } \\
\text { and city } \\
\text { hospitals }\end{array}$ & $\begin{array}{l}\text { Functional } \\
\text { impairment from } \\
\text { acute illness }\end{array}$ & 21 & $\begin{array}{l}\text { Age } \geq 65 \text {, at risk for admission to nursing home, reversible functional } \\
\text { problem, medically stable, not terminally ill or severely demented }\end{array}$ & 78.8 & $78 / 77$ \\
\hline $\begin{array}{l}\text { Cohen } 2002 \text {, } \\
\text { USA }^{2149}\end{array}$ & $\begin{array}{l}\text { Medical centre } \\
\text { (medical or } \\
\text { surgical ward) }\end{array}$ & $\begin{array}{l}\text { Acute medical or } \\
\text { surgical illnesses }\end{array}$ & NA & Age $\geq 65$, admitted from home, not terminally ill or severely demented & 74.2 & $694 / 694$ \\
\hline $\begin{array}{l}\text { Fleming 2004, } \\
\text { UK }^{44}\end{array}$ & $\begin{array}{l}\text { Community } \\
\text { hospital }\end{array}$ & $\begin{array}{l}\text { Acute medical or } \\
\text { orthopaedic } \\
\text { disorders }\end{array}$ & 35 & $\begin{array}{l}\text { Age } \geq 65 \text {, admitted from home, at risk for admission to nursing home, not } \\
\text { severely disabled or demented, medically stable }\end{array}$ & $81.5^{\star}$ & $81 / 84$ \\
\hline $\begin{array}{l}\text { Karppi } 1995, \\
\text { Finland }^{22}\end{array}$ & $\begin{array}{l}\text { Direct } \\
\text { admissions } \\
\text { from home }\end{array}$ & $\begin{array}{l}\text { Medical problems } \\
\text { with anticipated } \\
\text { benefit from } \\
\text { geriatric } \\
\text { intervention }\end{array}$ & $0 \dagger$ & $\begin{array}{l}\text { Age }>65 \text {, admitted from home, at risk for admission to nursing home, } \\
\text { reversible functional problem }\end{array}$ & 78.5 & $104 / 208$ \\
\hline $\begin{array}{l}\text { Rubenstein } \\
1984, \text { USA }^{5} 2930\end{array}$ & Medical centre & $\begin{array}{l}\text { Acute medical or } \\
\text { surgical illnesses }\end{array}$ & $\geq 7$ & $\begin{array}{l}\text { Age } \geq 65 \text { (all men), at risk for admission to nursing home, reversible } \\
\text { functional problem, not terminally ill or severely demented }\end{array}$ & 77.9 & $63 / 60$ \\
\hline $\begin{array}{l}\text { Saltvedt 2002, } \\
\text { Norway }^{14-16}\end{array}$ & $\begin{array}{l}\text { University } \\
\text { hospital }\end{array}$ & $\begin{array}{l}\text { Acute medical } \\
\text { illnesses }\end{array}$ & 0† & Age $\geq 75$, admitted from home, frail, not terminally ill or severely demented & 82.1 & $127 / 127$ \\
\hline $\begin{array}{l}\text { White 1994, } \\
\text { USA }^{27}\end{array}$ & $\begin{array}{l}\text { University } \\
\text { hospital }\end{array}$ & $\begin{array}{l}\text { Acute medical or } \\
\text { surgical illnesses }\end{array}$ & 17 & $\begin{array}{l}\text { Age }>65 \text {, at risk for admission to nursing home, reversible functional } \\
\text { problem, not severely demented, medically stable }\end{array}$ & 76.5 & $20 / 20$ \\
\hline $\begin{array}{l}\text { Young } 2007 \\
\text { UK }^{19} 1248\end{array}$ & $\begin{array}{l}\text { Community } \\
\text { hospital }\end{array}$ & $\begin{array}{l}\text { Acute medical or } \\
\text { surgical illnesses }\end{array}$ & $6^{*}$ & Age $>75$, reversible functional problem, medically stable & $86.0^{*}$ & $280 / 210$ \\
\hline \multicolumn{7}{|c|}{ Orthopaedic geriatric rehabilitation } \\
\hline $\begin{array}{l}\text { Cameron 1993, } \\
\text { Australia }^{11}\end{array}$ & $\begin{array}{l}\text { General } \\
\text { hospital }\end{array}$ & Acute hip fracturef & 2 & Age $\geq 65$ & 84.9 & $127 / 125$ \\
\hline $\begin{array}{l}\text { Gilchrist 1988, } \\
\text { UK }^{45}\end{array}$ & $\begin{array}{l}\text { University } \\
\text { hospital }\end{array}$ & Acute hip fracturef & 10 & Age $\geq 65$ (all women), expected prolonged hospital stay & 81.3 & $97 / 125$ \\
\hline $\begin{array}{l}\text { Huusko } 2002 \\
\text { Finland } \\
\end{array}$ & $\begin{array}{l}\text { Local health } \\
\text { centre hospital }\end{array}$ & Acute hip fracture $\ddagger$ & $\geq 1$ & $\begin{array}{l}\text { Age } \geq 65 \text {, admitted from home, good function before fracture, not } \\
\text { terminally ill or severely demented }\end{array}$ & 80.0 & $120 / 123$ \\
\hline $\begin{array}{l}\text { Kennie } 1988 \text {, } \\
\text { UK }^{23} 24\end{array}$ & $\begin{array}{l}\text { District } \\
\text { hospital }\end{array}$ & Acute hip fracture $\neq$ & 5 & Age $\geq 65$ (all women), expected length of hospital stay $>7$ days & $82^{*}$ & $54 / 54$ \\
\hline $\begin{array}{l}\text { Naglie } 2002 \text {, } \\
\text { Canada }^{13}\end{array}$ & $\begin{array}{l}\text { University } \\
\text { affiliated } \\
\text { hospital }\end{array}$ & Acute hip fracturef & $0 \dagger$ & $\begin{array}{l}\text { Age } \geq 70 \text {, admitted from home, good function before fracture, not } \\
\text { terminally ill }\end{array}$ & 84.2 & $141 / 139$ \\
\hline $\begin{array}{l}\text { Shyu } 2005 \text {, } \\
\text { Thailand }^{17}\end{array}$ & $\begin{array}{l}\text { Community } \\
\text { based medical } \\
\text { centre }\end{array}$ & Acute hip fracture $\neq$ & $0 \dagger$ & $\begin{array}{l}\text { Age } \geq 60 \text {, good function before fracture, not terminally ill or severely } \\
\text { demented }\end{array}$ & 77.6 & $72 / 87$ \\
\hline $\begin{array}{l}\text { Stenvall 2007, } \\
\text { Sweden }^{25}\end{array}$ & $\begin{array}{l}\text { University } \\
\text { hospital }\end{array}$ & Acute hip fracture $\ddagger$ & $0 \dagger$ & Age $>70$, good function before fracture & 82.2 & $102 / 97$ \\
\hline $\begin{array}{l}\text { Swanson 1998, } \\
\text { Australia }^{18}\end{array}$ & $\begin{array}{l}\text { University } \\
\text { hospital }\end{array}$ & Acute hip fracturef & $0 \dagger$ & Age $\geq 55$, admitted from home, good function before fracture & 78.1 & $38 / 33$ \\
\hline $\begin{array}{l}\text { Vidan 2005, } \\
\text { Spain }^{26}\end{array}$ & $\begin{array}{l}\text { University } \\
\text { hospital }\end{array}$ & Acute hip fracture & $0 \dagger$ & $\begin{array}{l}\text { Age }>65 \text {, admitted from home, good function before fracture, not terminally } \\
\text { ill }\end{array}$ & 81.9 & $155 / 164$ \\
\hline
\end{tabular}

$\mathrm{NA}=$ not available.

*Median.

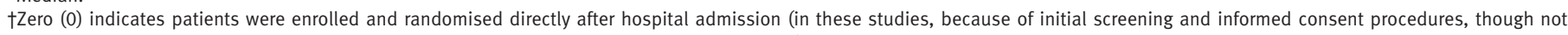
explicitly reported, patients might have had few inpatient hospital days before randomisation).

‡Most studies explicitly defined "acute hip fracture" as uncomplicated single sided proximal femur fracture (that is, no additional fractures, non-pathological fracture).

that staff assessing functional status and nursing home outcomes were blinded to the patients' allocation), and analyses by intention to treat (that is, participants were analysed within their randomised groups). ${ }^{31}$

\section{Statistical analysis}

For admission to a nursing home and mortality outcomes, we calculated relative risks with 95\% confidence intervals. Functional outcomes (primarily reported as means (SD) of the Barthel or Katz index) were converted to odds ratios and 95\% confidence intervals according to the method described by Chinn $^{32}$ and Hasselblad and Hedges. ${ }^{33}$ This method, used in previously published meta-analyses, ${ }^{434}$ is based on the fact that with logistic distributions and equal variances in the two treatment groups the log odds ratio corresponds to a constant multiplied by the standardised difference between means. 
Table 2 |Description of inpatient rehabilitation programmes specifically designed for geriatric patients in 17 included randomised controlled trials

\begin{tabular}{|c|c|c|c|c|c|c|c|c|}
\hline & \multirow[b]{2}{*}{$\begin{array}{l}\text { Programme } \\
\text { description }\end{array}$} & \multicolumn{2}{|c|}{ Structure of programme } & \multicolumn{5}{|c|}{ Process of programme } \\
\hline & & Location & Additional team members* & $\begin{array}{l}\text { Multidimen- } \\
\text { sional } \\
\text { geriatric } \\
\text { assessment }\end{array}$ & $\begin{array}{l}\text { Assignment } \\
\text { to therapy }\end{array}$ & $\begin{array}{l}\text { Intervention team } \\
\text { meetings for goal } \\
\text { setting }\end{array}$ & $\begin{array}{l}\text { Mean length of } \\
\text { hospital stay after } \\
\text { randomisation } \\
\text { (days) } \dagger\end{array}$ & $\begin{array}{c}\text { Follow-up } \\
\text { intervention after } \\
\text { hospital discharge }\end{array}$ \\
\hline \multicolumn{9}{|c|}{ General geriatric rehabilitation } \\
\hline $\begin{array}{l}\text { Apple- } \\
\text { gate } \\
1990\end{array}$ & $\begin{array}{l}\text { Geriatric } \\
\text { assessment unit }\end{array}$ & $\begin{array}{l}\text { Separate unit in } \\
\text { acute care hospital } \\
\text { complex }\end{array}$ & $\begin{array}{l}\text { Physiotherapist, occupational } \\
\text { therapist, psychologist, social } \\
\text { worker, speech therapist, } \\
\text { dietician }\end{array}$ & Yes & Yes & Weekly & 23.6 & No \\
\hline $\begin{array}{l}\text { Cohen } \\
2002\end{array}$ & $\begin{array}{l}\text { Geriatric } \\
\text { evaluation and } \\
\text { management unit }\end{array}$ & $\begin{array}{l}\text { Separate unit in } \\
\text { acute care medical } \\
\text { centre }\end{array}$ & $\begin{array}{l}\text { Physiotherapist, occupational } \\
\text { therapist, social worker, } \\
\text { dietician }\end{array}$ & Yes & Yes & Twice a week & 23.2 & $\begin{array}{l}\text { Yes (one } \\
\text { subsample: } \\
\text { outpatient careinVA } \\
\text { geriatric } \\
\text { programme) }\end{array}$ \\
\hline $\begin{array}{l}\text { Fleming } \\
2004\end{array}$ & $\begin{array}{l}\text { Care home } \\
\text { rehabilitation } \\
\text { services }\end{array}$ & $\begin{array}{l}\text { Separate } \\
\text { institution for } \\
\text { rehabilitation }\end{array}$ & $\begin{array}{l}\text { Social worker, occupational } \\
\text { therapist }\end{array}$ & No & No & No & 16.3 & No \\
\hline $\begin{array}{l}\text { Karppi } \\
1995\end{array}$ & $\begin{array}{l}\text { Geriatric inpatient } \\
\text { unit }\end{array}$ & $\begin{array}{l}\text { Separate unit in } \\
\text { acute care hospital }\end{array}$ & $\begin{array}{l}\text { Physiotherapist, occupational } \\
\text { therapist, social worker, } \\
\text { psychologist }\end{array}$ & Yes & NA & NA & 16.5 & No \\
\hline $\begin{array}{l}\text { Ruben- } \\
\text { stein } \\
1984 \\
\end{array}$ & $\begin{array}{l}\text { Geriatric } \\
\text { evaluation unit }\end{array}$ & $\begin{array}{l}\text { Separate unit in } \\
\text { acute care medical } \\
\text { centre }\end{array}$ & $\begin{array}{l}\text { Physiotherapist, occupational } \\
\text { therapist, social worker, } \\
\text { psychologist, dietician }\end{array}$ & Yes & Yes & Weekly & 85.1 & $\begin{array}{l}\text { Yes (continuing care } \\
\text { in geriatric } \\
\text { outpatient clinic) }\end{array}$ \\
\hline $\begin{array}{l}\text { Saltvedt } \\
2002\end{array}$ & $\begin{array}{l}\text { Geriatric } \\
\text { evaluation and } \\
\text { management unit }\end{array}$ & $\begin{array}{l}\text { Separate unit in } \\
\text { acute care hospital }\end{array}$ & $\begin{array}{l}\text { Physiotherapist, occupational } \\
\text { therapist }\end{array}$ & Yes & Yes & Twice a week & 21.2 & No \\
\hline $\begin{array}{l}\text { White } \\
1994\end{array}$ & $\begin{array}{l}\text { Multidisciplinary } \\
\text { geriatric care }\end{array}$ & $\begin{array}{l}\text { Separate unit in } \\
\text { acute care hospital }\end{array}$ & $\begin{array}{l}\text { Social worker, dietician, } \\
\text { occupational therapist, } \\
\text { pharmacist, physiotherapist }\end{array}$ & Yes & Yes & Yes & 7.7 & No \\
\hline $\begin{array}{l}\text { Young } \\
2007\end{array}$ & $\begin{array}{l}\text { Multidisciplinary } \\
\text { geriatric care }\end{array}$ & $\begin{array}{l}\text { Separate hospital } \\
\text { for rehabilitation }\end{array}$ & Physiotherapist, social worker & Yes & No & Yes & $22 \ddagger$ & No \\
\hline \multicolumn{9}{|c|}{ Orthopaedic geriatric rehabilitation } \\
\hline $\begin{array}{l}\text { Camer- } \\
\text { on } 1993\end{array}$ & $\begin{array}{l}\text { Accelerated } \\
\text { rehabilitation }\end{array}$ & $\begin{array}{l}\text { Combined acute/ } \\
\text { post-acute unit in } \\
\text { acute care hospital }\end{array}$ & $\begin{array}{l}\text { Physiotherapist, occupational } \\
\text { therapist, social worker }\end{array}$ & Yes & No & Yes & 19.5 & $\begin{array}{l}\text { Yes (day hospital or } \\
\text { physiotherapy) }\end{array}$ \\
\hline $\begin{array}{l}\text { Gilchrist } \\
1988\end{array}$ & $\begin{array}{l}\text { Orthopaedic } \\
\text { geriatric inpatient } \\
\text { care }\end{array}$ & $\begin{array}{l}\text { Separate hospital } \\
\text { for rehabilitation }\end{array}$ & $\begin{array}{l}\text { Orthopaedic surgeon, } \\
\text { physiotherapist, occupational } \\
\text { therapist, social worker }\end{array}$ & No & No & Weekly & 33.8 & No \\
\hline $\begin{array}{l}\text { Huusko } \\
2002\end{array}$ & $\begin{array}{l}\text { Intensive geriatric } \\
\text { rehabilitation }\end{array}$ & $\begin{array}{l}\text { Separate unit in } \\
\text { acute care hospital }\end{array}$ & $\begin{array}{l}\text { Physiotherapist, occupational } \\
\text { therapist, social worker, } \\
\text { psychologist }\end{array}$ & Yes & Yes & Weekly & 34 & $\begin{array}{l}\text { Yes (physiotherapy } \\
\text { for two months) }\end{array}$ \\
\hline $\begin{array}{l}\text { Kennie } \\
1988\end{array}$ & $\begin{array}{l}\text { Geriatric } \\
\text { rehabilitation }\end{array}$ & $\begin{array}{l}\text { Separate hospital } \\
\text { for rehabilitation }\end{array}$ & $\begin{array}{l}\text { Physiotherapist, occupational } \\
\text { therapist }\end{array}$ & Yes & No & Yes & $24 \ddagger$ & No \\
\hline $\begin{array}{l}\text { Naglie } \\
2002\end{array}$ & $\begin{array}{l}\text { Postoperative } \\
\text { multidisciplinary } \\
\text { care }\end{array}$ & $\begin{array}{l}\text { Separate unit in } \\
\text { acute care hospital }\end{array}$ & $\begin{array}{l}\text { Orthopaedic surgeon, } \\
\text { physiotherapist, occupational } \\
\text { therapist, social worker }\end{array}$ & Yes & No & No & 29.2 & No \\
\hline $\begin{array}{l}\text { Shyu } \\
2005\end{array}$ & $\begin{array}{l}\text { Multidisciplinary } \\
\text { intervention } \\
\text { programme }\end{array}$ & $\begin{array}{l}\text { Combined acute/ } \\
\text { post-acute unit in } \\
\text { acute care hospital }\end{array}$ & Physiotherapist & Yes & Yes & Yes & 10.1 & $\begin{array}{l}\text { Yes (geriatric nurse } \\
\text { coordination of } \\
\text { follow-up services } \\
\text { and clinic visits) }\end{array}$ \\
\hline $\begin{array}{l}\text { Stenvall } \\
2007\end{array}$ & $\begin{array}{l}\text { Multidisciplinary } \\
\text { postoperative } \\
\text { rehabilitation }\end{array}$ & $\begin{array}{l}\text { Combined acute/ } \\
\text { post-acute unit in } \\
\text { acute care hospital }\end{array}$ & $\begin{array}{l}\text { Physiotherapist, occupational } \\
\text { therapist, dietician }\end{array}$ & Yes & Yes & Yes & 30.0 & Yes \\
\hline $\begin{array}{l}\text { Swan- } \\
\text { son } \\
1998\end{array}$ & $\begin{array}{l}\text { Multidisciplinary } \\
\text { rehabilitation } \\
\text { intervention }\end{array}$ & $\begin{array}{l}\text { Combined acute/ } \\
\text { post-acute unit in } \\
\text { acute care hospital }\end{array}$ & $\begin{array}{l}\text { Orthopaedic surgeon, } \\
\text { physiotherapist, occupational } \\
\text { therapist, social worker }\end{array}$ & Yes & Yes & Weekly & 21.0 & $\begin{array}{l}\text { Yes (follow-up visit } \\
\text { one and six months } \\
\text { after discharge) }\end{array}$ \\
\hline $\begin{array}{l}\text { Vidan } \\
2005\end{array}$ & $\begin{array}{l}\text { Comprehensive } \\
\text { geriatric } \\
\text { intervention }\end{array}$ & $\begin{array}{l}\text { Combined acute/ } \\
\text { post-acute unit in } \\
\text { acute care hospital }\end{array}$ & $\begin{array}{l}\text { Social worker, rehabilitation } \\
\text { specialist }\end{array}$ & Yes & Yes & Weekly & $16 \ddagger$ & No \\
\hline
\end{tabular}

$\mathrm{NA}=$ not available.

*All teams included geriatrician and nurse.

tWeighted for sample size.

$\ddagger$ Median.

We used the "metan" command for Stata statistical software (version 10, StataCorp, College Station, TX) to conduct random effects meta-analyses. ${ }^{35}$
Heterogeneity between trials was measure with the $\mathrm{I}^{2}$ statistic, which indicates the proportion of the total variation in estimated effects caused by heterogeneity 
Table $3 \mid$ Reported outcomes in 17 included randomised controlled trials (listed alphabetically by type of rehabilitation) of inpatient rehabilitation programmes specifically designed for geriatric patients

\begin{tabular}{|c|c|c|c|c|c|c|c|}
\hline & \multicolumn{3}{|c|}{ Reported short term (at discharge) outcomes } & \multicolumn{4}{|c|}{ Reported longer term (at 3-12 month follow-up) outcomes } \\
\hline & $\begin{array}{c}\text { Functional } \\
\text { improvement } \\
\text { (instrument used)* }\end{array}$ & $\begin{array}{l}\text { Nursing home } \\
\text { admission }\end{array}$ & Mortality & $\begin{array}{c}\text { Functional } \\
\text { improvement } \\
\text { (instrument used)* }\end{array}$ & $\begin{array}{l}\text { Nursing home } \\
\text { admission }\end{array}$ & Mortality & $\begin{array}{l}\text { Length of follow-up } \\
\text { to outcome } \\
\text { measurement } \\
\text { (months) }\end{array}$ \\
\hline \multicolumn{8}{|c|}{ General geriatric rehabilitation } \\
\hline Applegate 1990 & No & Yes & No & ADL score & Yes & Yes & 12 \\
\hline Cohen 2002 & Katz index & No & No & Katz index & Yes & Yes & 12 \\
\hline Fleming 2004 & No & No & No & No & Yes & Yes & 12 \\
\hline Karppi 1995 & No & No & No & Katz index & Yes & Yes & $\begin{array}{l}3 \text { (function), } 12 \\
\text { (nursing home } \\
\text { admission, mortality) }\end{array}$ \\
\hline Rubenstein 1984 & No & Yes & Yes & $\begin{array}{l}\text { Personal self } \\
\text { maintenance scale }\end{array}$ & Yes & Yes & 12 \\
\hline Saltvedt 2002 & No & Yes & Yes & Barthel index & Yes & Yes & $\begin{array}{l}6 \text { (nursing home } \\
\text { admission), } 12 \\
\text { (function, mortality) }\end{array}$ \\
\hline White 1994 & Katz index & Yes & Yes & No & No & No & Discharge \\
\hline Young 2007 & Barthel index $†$ & No & Yes & Barthel index $†$ & Yes & Yes & 6 \\
\hline \multicolumn{8}{|c|}{ Orthopaedic geriatric rehabilitation } \\
\hline Cameron 1993 & No & No & No & Barthel index & Yes & No & 4 \\
\hline Gilchrist 1988 & No & Yes & Yes & No & No & Yes & 6 \\
\hline Huusko 2002 & No & Yes & Yes & No & Yes & Yes & 12 \\
\hline Kennie 1988 & Katz index & Yes & Yes & Katz index & Yes & Yes & 12 \\
\hline Naglie 2002 & No & Yes & Yes & Barthel index & Yes & Yes & 6 \\
\hline Shyu 2005 & Barthel index & Yes & Yes & Barthel index & Yes & Yes & 3 \\
\hline Stenvall 2007 & Katz index & Yes & Yes & Katz index & Yes & Yes & 12 \\
\hline Swanson 1998 & Barthel index & Yes & Yes & No & No & Yes & 6 \\
\hline Vidan 2005 & Katz index & No & Yes & Katz index & No & Yes & $\begin{array}{l}3 \text { (function), } 12 \\
\text { (mortality) }\end{array}$ \\
\hline
\end{tabular}

$A D L=$ activities of daily living.

*See methods for references of functional status measures.

†Functional status outcomes reported for only one study site (intervention group $n=79$, control group $n=141$ ).

between trials rather than chance. ${ }^{36} \mathrm{We}$ also calculated the $\mathrm{P}$ value for the $\chi^{2}$ test of heterogeneity. We explored the extent to which one or more study characteristics explained heterogeneity between trials using random effects meta-regression, with the Stata "metareg" command. According to an a priori statistical analysis plan we considered type of intervention programme (general geriatric/orthopaedic), mean (or median) age of total study population $(\leq 80 v>80)$, length of hospital stay after randomisation in the intervention group $(\leq 21$ $v>21$ days), outpatient follow-up therapy after the trial for patients in the intervention group (yes/no), length of follow-up for outcome evaluation ( $\leq 6 v>6$ months), quality of the intervention programme (use of multidimensional geriatric assessment and assignment of patients), and methodological trial quality (concealment of allocation, blinding of outcome assessor, and analysis by intention to treat). For all outcomes we included the selected variables in meta-regression models and conducted random effects meta-analyses within each subgroup. We used funnel plots and Begg and Egger bias tests to check for small study effects. ${ }^{37-41}$ Finally, we calculated the numbers needed to treat (NNT) to prevent one admission to a nursing home and death. ${ }^{42}$

\section{RESULTS}

Identification of eligible trials

We identified 932 potentially relevant publications. Twenty seven articles ${ }^{511-1921-3043-49}$ describing 17 trials met the predefined inclusion criteria and were included in our meta-analysis (fig 1).

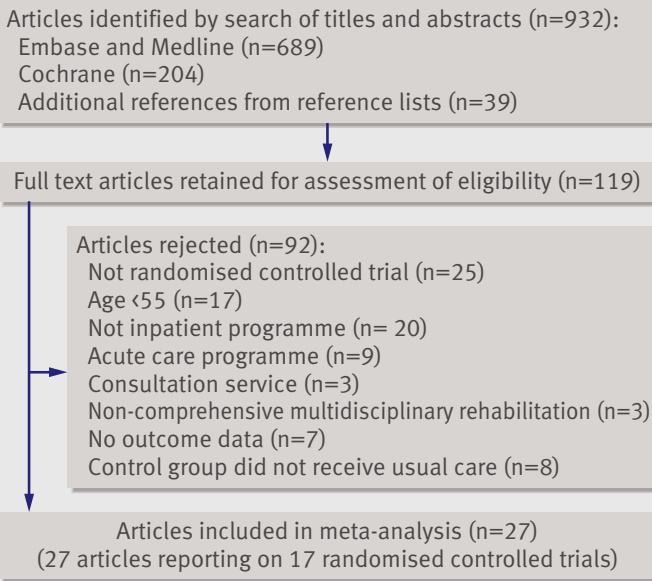

Fig 1| Flow of papers through study 


\section{Characteristics of trials, participants, and intervention programmes}

Tables 1 and 2 describe the 17 trials and corresponding inpatient rehabilitation intervention programmes. We found eight trials on general geriatric rehabilitation $^{51214-1619212227-3043444849}$ and nine on orthopaedic geriatric rehabilitation after hip fracture. ${ }^{1113171823-2645-47}$ No trials reporting effects of rehabilitation programmes in other specialties (such as orthopaedic for other indications, neurological, cardiac, or pulmonary) met the inclusion criteria (randomised controlled trials of neurological rehabilitation, for example, often had no lower age cut off and included younger patients). In the 17 included trials, 4780 people were allocated to intervention $(n=2353)$ or control ( $\mathrm{n}=2427)$ groups. Mean (or median) age of participants ranged from 74.2 to 86.0 (table 1). Type and duration of hospital care before the trial, reason for the initial hospital admission, and criteria for the selection of patients (such as age criteria, functional criteria) varied between studies (table 1). Table 2 shows the main structural (location, personnel) and process (based on WHO criteria) characteristics.

Table 3 gives details of the reported outcomes. At discharge eight $(47 \%)$ of the 17 trials reported functional status in a way that we could extract and compare results between trials, $10(59 \%)$ reported admissions to nursing homes, and $12(71 \%)$ reported mortality. At the end of follow-up $12(71 \%)$ trials reported on functional status, $13(76 \%)$ on admission to nursing homes, and $15(88 \%)$ on mortality (table 3). Length of follow-up for outcomes ranged from discharge to 12 months. Funnel plots and bias tests indicated little evidence of publication bias.

\section{Short term effects at discharge}

At discharge, heterogeneity tests for the three outcomes were not significant $\left(\mathrm{I}^{2}=38.4 \%, \mathrm{P}=0.12\right.$, for functional improvement; $\mathrm{I}^{2}=14.6 \%, \mathrm{P}=0.31$, for admission to nursing home; and $\mathrm{I}^{2}=0.0 \%, \mathrm{P}=0.56$, for mortality) (figs 2-4). Overall, trials had a short term beneficial effect on all outcomes (combined odds ratio 1.75

\begin{tabular}{|c|c|c|c|c|c|c|}
\hline \multirow[b]{2}{*}{ Study characteristics } & \multicolumn{3}{|c|}{ Short term (at discharge) } & \multicolumn{3}{|c|}{ Longer term (at 3-12 month follow-up) } \\
\hline & $\begin{array}{l}\text { Functional } \\
\text { improvement }\end{array}$ & $\begin{array}{l}\text { Nursing home } \\
\text { admission }\end{array}$ & Mortality & $\begin{array}{l}\text { Functional } \\
\text { improvement }\end{array}$ & $\begin{array}{l}\text { Nursing home } \\
\text { admission }\end{array}$ & Mortality \\
\hline \multicolumn{7}{|c|}{ Type of intervention programme: } \\
\hline $\begin{array}{l}\text { General geriatric } \\
\text { rehabilitation }\end{array}$ & 1.34 (1.12 to 1.60$)$ & $0.53(0.33$ to 0.86$)$ & $0.76(0.54$ to 1.06$)$ & $1.02(0.86$ to 1.21$)$ & $0.90(0.71$ to 1.13$)$ & 0.88 (0.75 to 1.04$)$ \\
\hline $\begin{array}{l}\text { Orthopaedic geriatric } \\
\text { rehabilitation }\end{array}$ & $\begin{array}{c}2.33 \text { (1.62 to } 3.34), \\
P=0.04^{*}\end{array}$ & $0.72(0.56$ to 0.91$)$ & $0.66(0.42$ to 1.04$)$ & $\begin{array}{c}1.79(1.24 \text { to } 2.60), \\
P=0.01^{*}\end{array}$ & $0.79(0.61$ to 1.02$)$ & 0.77 (0.61 to 0.96$)$ \\
\hline \multicolumn{7}{|c|}{ Mean age of study population (years): } \\
\hline$\leq 80$ & $1.88(1.19$ to 2.97$)$ & $0.42(0.27$ to 0.64$)$ & $1.01(0.47$ to 2.16$)$ & $1.26(0.87$ to 1.82$)$ & $0.82(0.58$ to 1.16$)$ & $0.85(0.62$ to 1.16$)$ \\
\hline$>80$ & $1.74(1.05$ to 2.88$)$ & $\begin{array}{c}0.75 \text { (0.58 to } 0.96) \\
\mathrm{P}=0.045^{\star}\end{array}$ & 0.68 (0.50 to 0.92$)$ & $1.45(1.08$ to 1.94$)$ & $0.88(0.72$ to 1.07$)$ & $0.82(0.70$ to 0.95$)$ \\
\hline \multicolumn{7}{|c|}{ Intervention programme: length of hospital stay after randomisation in intervention group: } \\
\hline$\leq 21$ days & $2.38(1.53$ to 3.70$)$ & 0.67 (0.42 to 1.09$)$ & $0.59(0.29$ to 1.22$)$ & $1.43(1.00$ to 2.05$)$ & $0.99(0.69$ to 1.42$)$ & $0.84(0.67$ to 1.05$)$ \\
\hline$>21$ days & $1.52(1.08$ to 2.13$)$ & 0.61 (0.45 to 0.83$)$ & $0.76(0.56$ to 1.04$)$ & $1.30(0.95$ to 1.78$)$ & 0.81 (0.67 to 0.98$)$ & 0.85 (0.73 to 0.99$)$ \\
\hline \multicolumn{7}{|c|}{ Outpatient follow-up after inpatient rehabilitation for patients in intervention group: } \\
\hline Yes & - & - & - & 1.49 (0.93 to 2.39$)$ & $0.76(0.64$ to 0.92$)$ & $0.84(0.61$ to 1.16$)$ \\
\hline No/NR & - & - & - & $1.29(1.00$ to 1.66$)$ & $0.88(0.69$ to 1.12$)$ & $0.82(0.71$ to 0.96$)$ \\
\hline \multicolumn{7}{|c|}{ Length of follow-up for outcome evaluation (months): } \\
\hline$\leq 6$ & - & - & - & 1.44 (0.94 to 2.21$)$ & $0.82(0.67$ to 1.00$)$ & $0.83(0.66$ to 1.05$)$ \\
\hline$>6$ & - & - & - & $1.32(0.99$ to 1.76$)$ & 0.90 (0.68 to 1.19$)$ & 0.85 (0.73 to 0.99$)$ \\
\hline \multicolumn{7}{|c|}{ Intervention programme quality: intervention included initial multidimensional geriatric assessment: } \\
\hline Yes & 1.75 (1.31 to 2.35$)$ & $0.60(0.46$ to 0.78$)$ & 0.75 (0.57 to 0.99) & $1.36(1.07$ to 1.71$)$ & $0.81(0.69$ to 0.94$)$ & $0.86(0.77$ to 0.97$)$ \\
\hline No/NR & NA & 0.84 (0.54 to 1.29$)$ & $0.40(0.13$ to 1.18$)$ & NA & $1.23(0.75$ to 2.02$)$ & $0.90(0.61$ to 1.33$)$ \\
\hline \multicolumn{7}{|c|}{ Intervention programme quality: intervention included patient assignment (to therapies and goal setting): } \\
\hline Yes & $1.81(1.23$ to 2.67$)$ & $0.54(0.37$ to 0.78$)$ & 0.68 (0.39 to 1.19$)$ & 1.32 (0.90 to 1.92$)$ & $0.88(0.62$ to 1.26$)$ & $0.83(0.68$ to 1.00$)$ \\
\hline No/NR & $1.80(0.96$ to 3.37$)$ & $0.71(0.53$ to 0.94$)$ & $0.74(0.53$ to 1.03$)$ & $1.40(1.02$ to 1.90$)$ & $0.86(0.71$ to 1.04$)$ & $0.85(0.71$ to 1.02$)$ \\
\hline \multicolumn{7}{|c|}{ Methodological trial quality: concealed randomisation: } \\
\hline Yes & $1.61(1.21$ to 2.13$)$ & $0.61(0.45$ to 0.83$)$ & 0.75 (0.56 to 1.02$)$ & $1.45(1.02$ to 2.06$)$ & $0.78(0.68$ to 0.91$)$ & 0.90 (0.79 to 1.03$)$ \\
\hline No/NR & 2.91 (1.36 to 6.24) & $0.66(0.40$ to 1.07$)$ & $0.54(0.24$ to 1.22$)$ & 1.29 (0.99 to 1.68$)$ & $1.05(0.72$ to 1.54$)$ & $0.77(0.60$ to 0.98$)$ \\
\hline \multicolumn{7}{|c|}{ Methodological trial quality: data assessment by an independent assessor: } \\
\hline Yes & $1.39(1.17$ to 1.65$)$ & 0.59 (0.33 to 1.06$)$ & $0.63(0.31$ to 1.26$)$ & 1.34 (0.97 to 1.85$)$ & 0.81 (0.68 to 0.95$)$ & $0.92(0.80$ to 1.06$)$ \\
\hline No/NR & 2.43 (1.47 to 4.00$)$ & 0.63 (0.47 to 0.85$)$ & 0.72 (0.48 to 1.07$)$ & 1.40 (0.97 to 2.03$)$ & 0.93 (0.66 to 1.30$)$ & $0.76(0.62$ to 0.93$)$ \\
\hline
\end{tabular}

$\mathrm{NR}=$ not reported, $\mathrm{NA}=$ not applicable (no studies in this category).

${ }^{*}$ Exact $P$ values for significant results in meta-regression analyses ( $\left.P<0.05\right)$. If result of meta-regression was not significant ( $\left.P \geq 0.05\right)$, no $P$ value is listed. 
(1.31 to 2.35$)$ for function, relative risk 0.64 ( 0.51 to 0.81 ) for nursing home admission, relative risk 0.72 (0.55 to 0.95$)$ for morality). Comparisons of short term outcomes between trials according to characteristics of the programme or patients showed two significant differences in effect. Specifically in metaregression analyses (table 4), there was greater functional improvement for orthopaedic compared with general geriatric rehabilitation programmes (odds ratio 2.33 (1.62 to 3.34 ) v 1.34 (1.12 to 1.60$), \mathrm{P}=0.04$ ); and a larger reduction in admissions to nursing homes in trials with younger patients (mean age of study population $\leq 80)$ than with older patients $(>80)$ (relative risk $0.42(0.27$ to 0.64$) v 0.75$ (0.58 to 0.96$), \mathrm{P}=0.045)$.

\section{Longer term effects at end of follow-up}

At the end of follow-up, results on function were heterogeneous $\left(\mathrm{I}^{2}=51.4 \%, \mathrm{P}=0.02\right)$. Overall results for Cohn 2002

\section{Effects at hospital discharge}

General geriatric rehabilitation

White 1994

Young 2007

Subtotal: $\left.\right|^{2}=0.0 \%, P=0.821$

Orthopaedic geriatric rehabilitation Kennie 1988

Shyu 2005

Stenwal 2007

Swanson 1998

Vidan 2005

Subtotal: $\left.\right|^{2}=0.0 \%, P=0.428$

Overall: $I^{2}=38.4 \%, P=0.123$

\section{Effects at 3-12 month follow-up}

General geriatric rehabilitation Applegate 1990

Cohen 2002

Karppi 1995

Rubenstein 1984

Saltvedt 2002

Young 2007

Subtotal: $I^{2}=0.0 \%, P=0.949$

Orthopaedic geriatric rehabilitation Cameron 1993

Kennie 1988

Naglie 2002

Shyu 2005

Stenvall 2007

Vidan 2005

Subtotal: $\left.\right|^{2}=53.5 \%, P=0.057$

Overall: $\left.\right|^{2}=51.4 \%, P=0.020$

Odds ratio ( $95 \% \mathrm{Cl})$

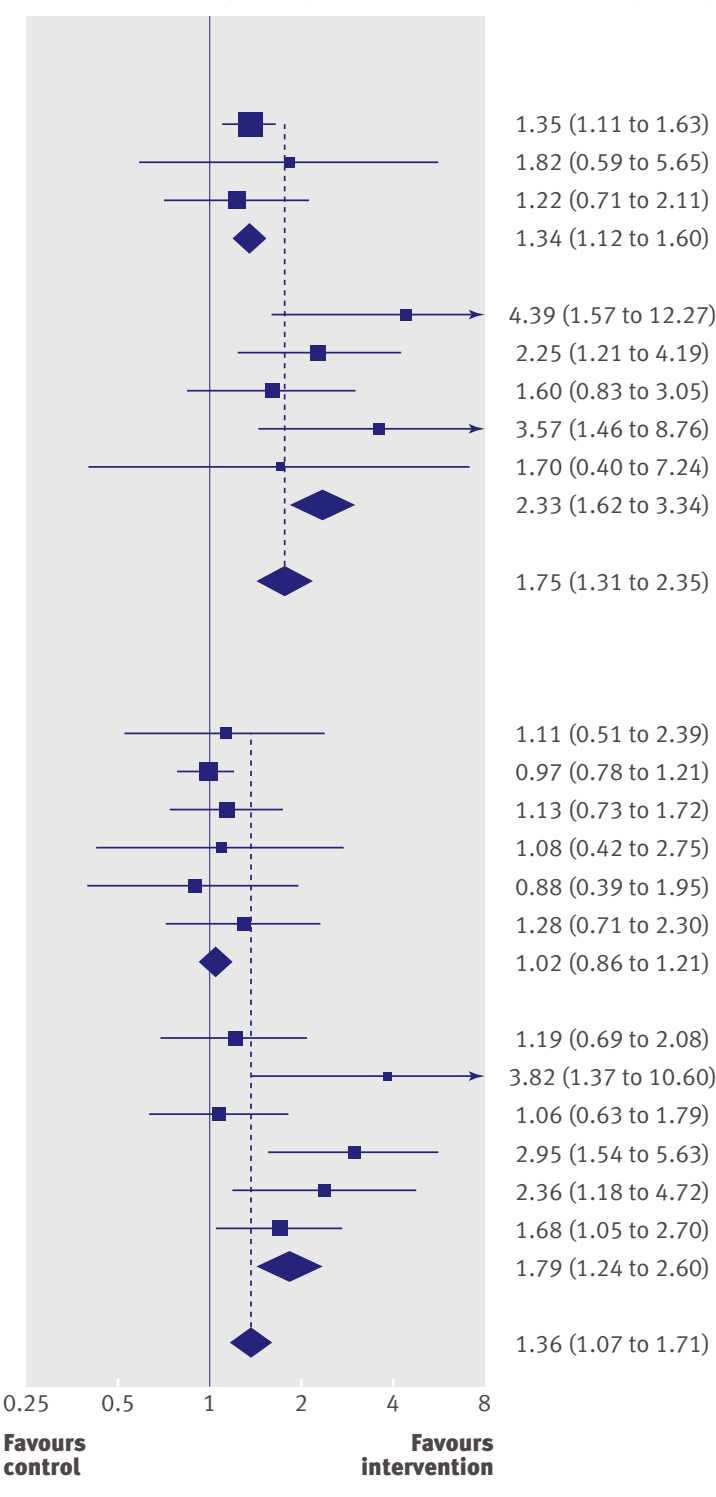

Fig 2 | Effect of inpatient rehabilitation specifically designed for geriatric patients on functional improvement at hospital discharge and at follow-up admissions to nursing homes and mortality were not heterogeneous $\left(\mathrm{I}^{2}=22.6 \%, \quad \mathrm{P}=0.22\right.$, and $\mathrm{I}^{2}=0.0 \%$, $\mathrm{P}=0.60$, respectively) (figs 2-4). Meta-analyses of longer term effects indicated an overall significant favourable effect of the intervention on all outcomes at the end of follow-up (combined odds ratio 1.36 (1.07 to 1.71$)$ for function, relative risk 0.84 (0.72 to 0.99 ) for nursing home admission, relative risk 0.87 (0.77 to 0.97 ) for mortality). Stratified effects of functional improvement at end of follow-up significantly differed by intervention type (odds ratio 1.02 (0.86 to 1.21) for general $v 1.79$ (1.24 to 2.60) for orthopaedic, $\mathrm{P}=0.01$ ). All other longer term effects (table 4) were similar across study characteristics hypothesised to influence longer term effects and not significant in meta-regression $(\mathrm{P}>0.05)$. In addition, heterogeneity between studies also varied by intervention type for both functional status and admissions to nursing homes (function: $\mathrm{I}^{2}=0.0 \%, \mathrm{P}=0.95$, for general $v$ $\mathrm{I}^{2}=53.5 \%, \mathrm{P}=0.06$, for orthopaedic; nursing home admission: $\mathrm{I}^{2}=42.8 \%, \mathrm{P}=0.11$, for general $v \mathrm{I}^{2}=0.0 \%$, $\mathrm{P}=0.43$, for orthopaedic).

The proportion of people in control groups admitted to a nursing home varied between $10 \%$ and $30 \%$ in most studies, resulting in a number needed to treat between 9 and 28 to avoid one admission to a nursing home at hospital discharge, and between 21 and 63 to avoid one admission to a nursing home at follow-up. The number needed to treat to prevent on death at one year follow-up was 38, assuming a $20 \%$ one year mortality rate among controls.

\section{Measures of intervention programme quality and methodological quality of trials}

Of the 17 included trials, $15(88 \%)$ reported that they included multidimensional geriatric assessment as part of the intervention, and 10 (59\%) reported on assignment as part of the intervention (table 2). Overall, 10 $(59 \%)$ trials reported adequate concealment of allocation, ${ }^{13-17} 1921232527284647$ seven (41\%) blinded the independent outcome assessors, ${ }^{13} 171921262844$ and all reported intention to treat. In meta-regression analyses, there were no significant differences in effects related to any of these quality criteria.

\section{Impact of intervention programmes on healthcare}

Compared with patients in control groups, the weighted mean length of hospital stay after randomisation was longer in patients allocated to general geriatric rehabilitation (24.5 $v 15.1$ days) and shorter in those allocated to orthopaedic rehabilitation (24.6 $v 28$. 9 days). Six out of 17 trials reported on readmission to hospital after rehabilitation. ${ }^{514-172527293044}$ All six reported a lower or equal rate in intervention patients, but the overall range across the six trials was similar in intervention (21-51\%) and control patients (31-55\%). Six trials reported total costs of inpatient geriatric rehabilitation programmes. ${ }^{192729434749}$ Total programme costs adjusted for years survived or living in the community differed between the trials: one trial showed no cost difference between intervention and usual care,$^{19}$ 


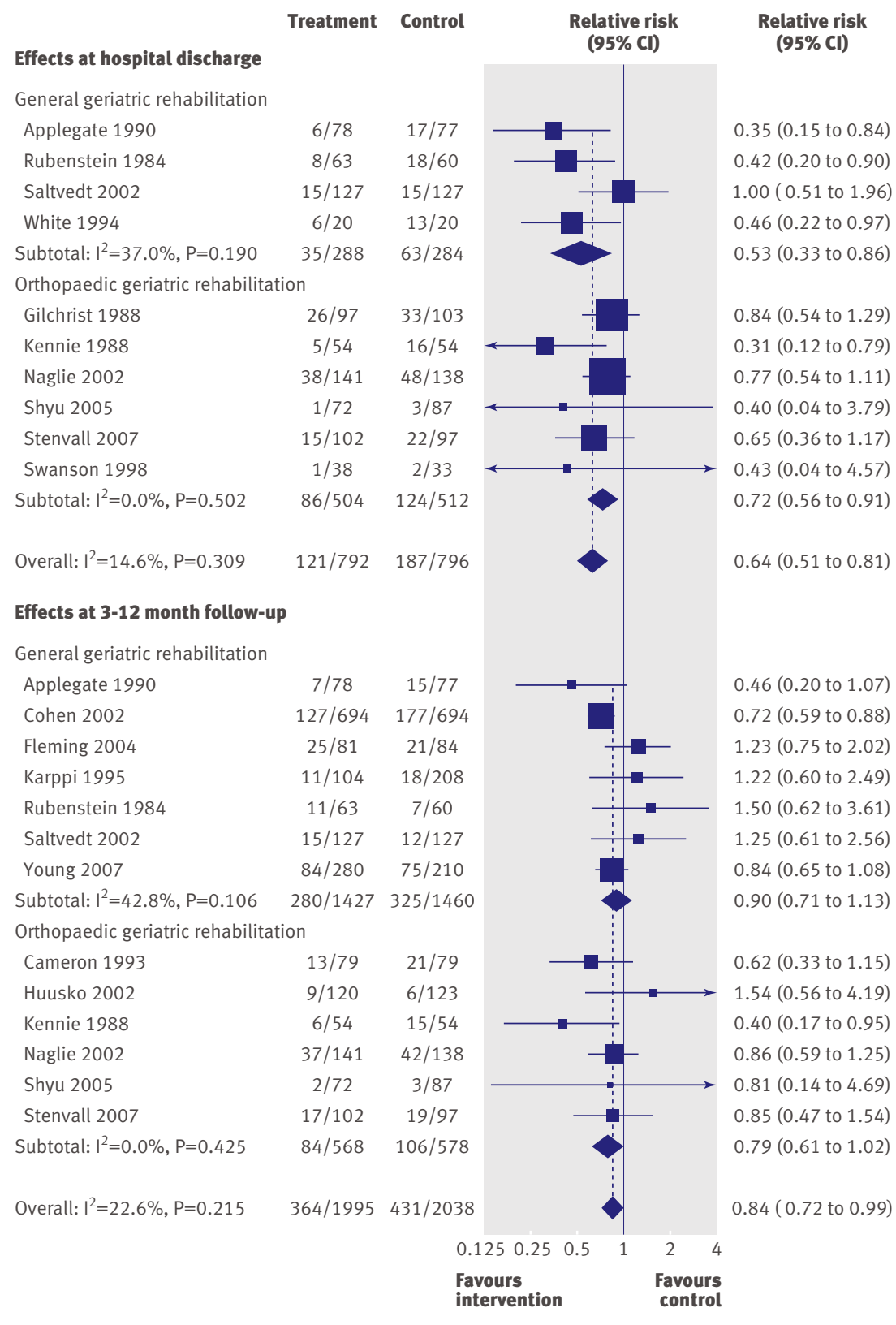

Fig 3 | Effect of inpatient rehabilitation specifically designed for geriatric patients on admissions to nursing homes at hospital discharge and at follow-up

and five trials showed cost differences that were not significant. Three trials showed cost savings in the intervention group, ${ }^{272949}$ and two trials showed the opposite. $^{4347}$

\section{DISCUSSION}

This systematic review and meta-analysis of inpatient rehabilitation specifically designed for geriatric patients showed beneficial effects over usual care for functional improvement, preventing admissions to nursing homes, and reducing mortality. For all outcomes inpatient rehabilitation showed a short term effect after discharge as well as a less pronounced longer term effect at the end of follow-up. Multiple stratified analyses according to characteristics of the programme, patients, and quality of the study showed only two significant differences in effects between study subgroups: orthopaedic intervention programmes were more likely to be associated with functional improvement, and study populations with a younger mean age showed a more beneficial effect for the nursing home outcome than those with a higher mean age. Given our extensive search strategy we are confident that we have identified all published randomised controlled trials meeting our inclusion criteria so that we included only trials examining programmes in accordance with WHO's definition of rehabilitation and the ICF framework.

\section{Comparison with other studies}

To our knowledge this is the first study to show the effects of inpatient rehabilitation specifically designed for older adults. Earlier meta-analyses found favourable effects of geriatric rehabilitation compared with usual hospital care but grouped randomised controlled trials of inpatient rehabilitation together with those of acute geriatric units. ${ }^{5051}$ One exception was a recent study that analysed the separate effects of acute geriatric units but not those of inpatient rehabilitation. ${ }^{6}$ Our meta-analysis now provides evidence for independent favourable effects of inpatient geriatric rehabilitation on function, admission to nursing homes, and mortality.

Our stringent definition of rehabilitation contrasts with recent literature on the topic of geriatric rehabilitation, ${ }^{5253}$ where the term "rehabilitation" is used in various ways. For example, Gill et al performed a randomised controlled trial in an outpatient setting where a physiotherapy intervention (labelled rehabilitation) was compared with educational interventions. ${ }^{52}$ Wells et al used a definition of rehabilitation similar to ours but did not emphasise the assignment procedure with goal setting. ${ }^{53}$

Our findings add to the existing literature evaluating the best methods of treating geriatric patients. They are in concordance with earlier meta-analyses showing favourable effects of other types of inpatient and outpatient programmes specifically designed for geriatric patients. ${ }^{4505154}$ They are also in concordance with criteria of successful geriatric programmes identified in earlier meta-analyses. These analyses showed that programmes were more successful if they used multidimensional geriatric assessment as a basis for problem identification, ${ }^{4}$ they had a geriatric team that formulated recommendations and controlled implementation of the recommendations, ${ }^{51}$ and inpatient geriatric care was combined with outpatient geriatric followup. ${ }^{51}$ These three criteria were at least in part met by the studies included in our meta-analysis.

\section{Limitations}

Given the number of stratified analyses, the more favourable effects in orthopaedic intervention programmes or populations with a younger mean age might be due to chance. Furthermore, given the limited number of included studies we might have missed true 
differences between study subgroups. For example, the difference in effects at discharge between trials by length of hospital stay after randomisation did not reach significance but might reflect real differences. Another limitation was introduced by differences in length of stay after randomisation in intervention compared with control patients, potentially influencing the comparability of outcome data measured at hospital discharge. There was heterogeneity at the end of follow-up for the pooled effect of orthopaedic intervention programmes on functional status and general geriatric intervention programmes on admissions to

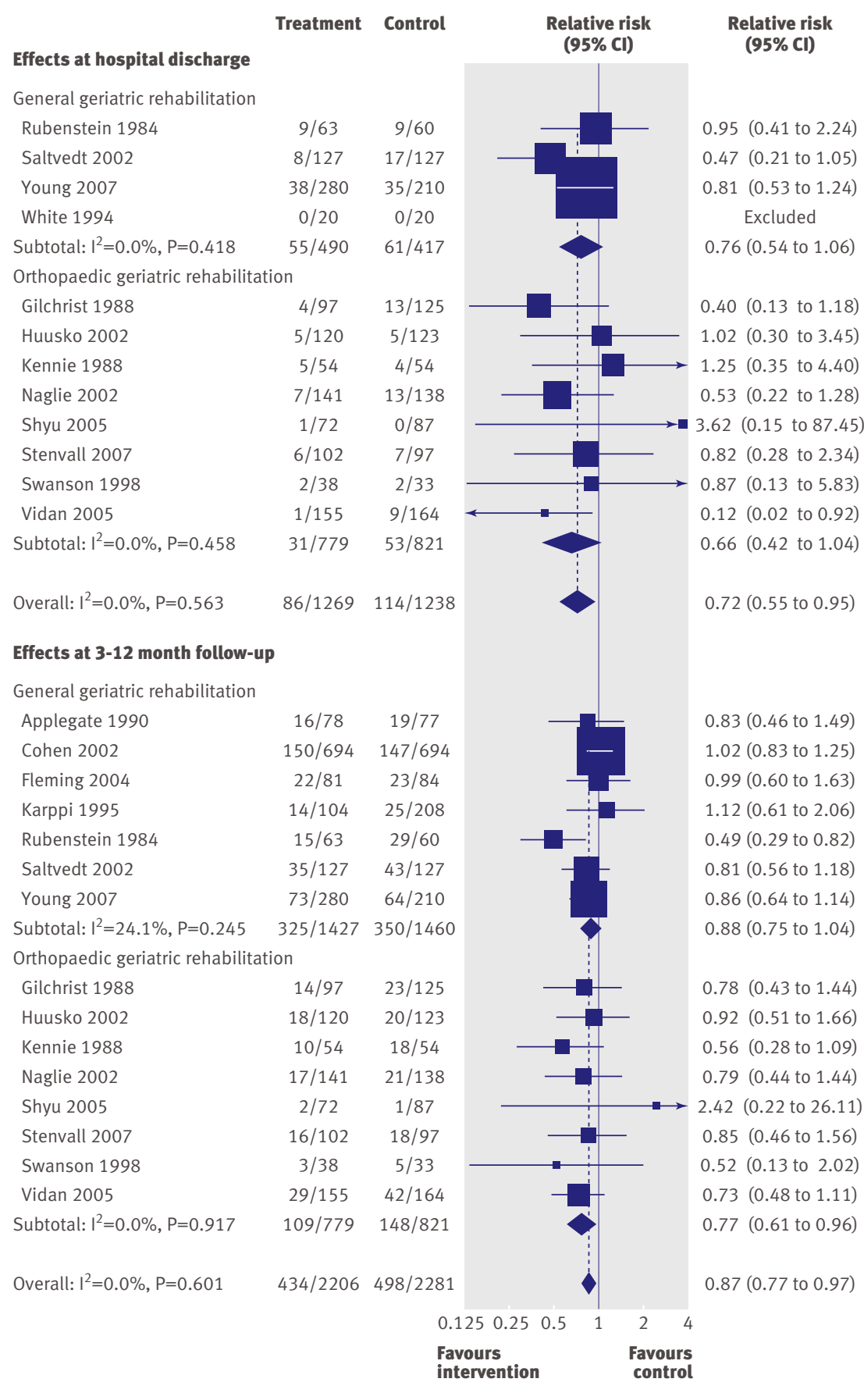

Fig 4 | Effect of inpatient rehabilitation specifically designed for geriatric patients on mortality at hospital discharge and at follow-up nursing homes. Therefore, these pooled effects should be interpreted with caution because the true differences in effects between studies might be due to uncharacterised or unexplained underlying factors or the variability of outcome measures on functional status. Finally, we used aggregate data rather than data on individual patients for the meta-regression analyses. As a result we cannot exclude confounding of the analyses, and potentially key associations might have been missed, underestimated, or reversed.

Longer term effects of rehabilitation for all outcomes seemed to be less pronounced than short term effects. This might in part be explained by variable and potentially suboptimal treatments of intervention patients after hospital discharge. Additionally, outpatient treatments after the intervention were seldom described, making interpretation of their potential influences on longer term effects impracticable.

\section{Implications}

Surprisingly, among the trials included in this metaanalysis, we found only two types of geriatric rehabilitation programmes: general and orthopaedic for hip fracture. No rehabilitation study in any other clinical specialty (such as cardiac, stroke, or pulmonary) met our inclusion criteria of being designed specifically for the care of geriatric patients, thus indicating a need for programme development and research for other types of rehabilitation. Some other type of inpatient rehabilitation might produce similar favourable effects if they are specifically designed for geriatric patients and might actually change clinical practice in the future.

Difference in effects between subtypes of geriatric rehabilitation programmes is a potentially important focus for research with relevant practice implications. Firstly, further analysis should determine whether orthopaedic rehabilitation programmes truly reduce length of inpatient hospital stay and thereby potentially reduce healthcare costs and at the same time improve patients' outcomes. If confirmed, this type of intervention might exhibit the rare combination of an intervention that reduced healthcare costs and improves patients' outcomes, giving it a high priority for implementation in practice. In contrast, general geriatric rehabilitation programmes seemed to increase length of inpatient stay. In these programmes intervention patients were probably offered an additional inpatient rehabilitation programme, resulting in initially increased healthcare costs in contrast with control patients who were probably discharged early to a long term care setting. Further research should determine cost effectiveness based on a longer term time frame and should define criteria for targeting patients in clinical practice. Finally, research might find answers for other types of programmes that, until now, have not been adapted and tested for the specific needs of older patients.

Inpatient rehabilitation specifically designed for geriatric patients is a resource intensive, and therefore expensive, component of health care. We considered it important to assess the impact on health care of 


\section{WHAT IS ALREADY KNOWN ON THIS TOPIC}

Inpatient rehabilitation programmes specifically designed for patients with cardiac, neurological, pulmonary, or musculoskeletal problems have been shown to improve outcomes

Older patients admitted to acute care hospitals are at increased risk for decline in functional status and admission to nursing homes

\section{WHAT THIS STUDY ADDS}

Inpatient geriatric rehabilitation programmes specifically designed for older people show sustained effects on improving functional status and reducing admissions to nursing homes and mortality

Such programmes might increase or decrease the overall length of hospital stay, depending on type and concept of the programme

Reduction in admissions to nursing homes might result in cost savings or offset additional costs of the initial inpatient rehabilitation unclear, as does the efficacy of characteristics of individual interventions and the impact on health care of such programmes (that is, cost-benefit and re-admission rates). Although we tried to clarify these questions with our work, the paucity of available evidence emphasises the need for further research to address these important questions.

Contributors: SB and AES were responsible for study concept, study design, data collection, data analysis, data interpretation, and preparation of manuscript. CF was responsible for study design, data collection, data analysis, data interpretation, preparation of manuscript. AH was responsible for data collection, data analysis, and preparation of manuscript. ME was responsible for data interpretation, and preparation of manuscript. KMC was responsible for study concept, data analysis, data interpretation, and preparation of manuscript. AES is guarantor. Funding: SB was supported by a Forschungskolleg Geriatrie Grant from the Robert Bosch Foundation, Stuttgart, Germany (grant No 32.5.1141.0021.0).

Competing interests: All authors have completed the Unified Competing Interest form at www.icmje.org/coi_disclosure.pdf (available on request from the corresponding author) and declare that (1) SB received financia support from the Robert Bosch Foundation (see above) for the submitted work, and no other support was received for the submitted work; (2) the have no relationships with companies that might have an interest in the submitted work in the previous 3 years; (3) their spouses, partners, or children have no financial relationships that may be relevant to the submitted work; and (4) they have no non-financial interests that may be relevant to the submitted work.

Ethical approval: Not required.

Data sharing: Funnel plots and a list of excluded trials are available from corresponding author on request.

1 WHO Expert Committee on Disability Prevention and Rehabilitation Disability prevention and rehabilitation. World Health Organ Tech Rep Ser 1981;668:1-39.

2 Gaugler JE, Duval S, Anderson KA, Kane RL. Predicting nursing home admission in the US: a meta-analysis. BMC Geriatr 2007:7:13.

3 Ottenbacher KJ, Smith PM, Illig SB, Linn RT, Ostir GV, Granger CV. Trends in length of stay, living setting, functional outcome, and mortality following medical rehabilitation. JAMA 2004:292:1687-95.

4 Huss A, Stuck AE, Rubenstein LZ, Egger M, Clough-Gorr KM. Multidimensional preventive home visit programs for communitydwelling older adults: a systematic review and meta-analysis of randomized controlled trials. J Gerontol A Biol Sci Med Sci 2008;63:298-307.

5 Rubenstein I7, Josephson KR, Harker JO, Miller DK, Wieland D. The Sepulveda GEU Study revisited: long-term outcomes, use of services, and costs. Aging (Milano) 1995;7:212-7.

6 Baztan JJ, Suarez-Garcia FM, Lopez-Arrieta J, Rodriguez-Manas L, Rodriguez-Artalejo F. Effectiveness of acute geriatric units on functional decline, living at home, and case fatality among older patients admitted to hospital for acute medical disorders: metaanalysis. BMJ 2009;338:b50.

7 Stucki G, Ewert T, Cieza A. Value and application of the ICF in rehabilitation medicine. Disabil Rehabil 2003;25:628-34.

8 Stucki G, Cieza A, Melvin I. The international classification of functioning, disability and health (ICF): a unifying model for the conceptual description of the rehabilitation strategy. J Rehabil Med 2007:39:279-85.

9 Stucki G, Melvin J. The international classification of functioning, disability and health: a unifying model for the conceptual description of physical and rehabilitation medicine. / Rehabil Med 2007;39:286-92.

10 Mahoney Fl, Barthel DW. Functional evaluation: the Barthel index Md State Med I 1965;14:61-5.

11 Cameron ID, Lyle DM, Quine S. Accelerated rehabilitation after proximal femoral fracture: a randomized controlled trial. Disabil Rehabil 1993;15:29-34.

12 Green J, Young J, Forster A, Mallinder K, Bogle S, Lowson K, et al. Effects of locality based community hospital care on independence in older people needing rehabilitation: randomised controlled trial. BMJ 2005;331:317-22.

13 Naglie G, Tansey C, Kirkland IL, Ogilvie-Harris DJ, Detsky AS, Etchells $\mathrm{E}$, et al. Interdisciplinary inpatient care for elderly people with hip fracture: a randomized controlled trial. CMAJ 2002;167:25-32.

14 Saltvedt I, Mo ES, Fayers P, Kaasa S, Sletvold O. Reduced mortality in treating acutely sick, frail older patients in a geriatric evaluation and 
management unit. A prospective randomized trial. / Am Geriatr Soc 2002;50:792-8

15 Saltvedt I, Saltnes T, Mo ES, Fayers P, Kaasa S, Sletvold O. Acute geriatric intervention increases the number of patients able to live at home. A prospective randomized study. Aging Clin Exp Res 2004;16:300-6.

16 Saltvedt I, Jordhoy M, Opdahl Mo ES, Fayers P, Kaasa S, Sletvold O. Randomised trial of in-hospital geriatric intervention: impact on function and morale. Gerontology 2006;52:223-30.

17 Shyu YI, Liang J, Wu CC, Su JY, Cheng HS, Chou SW, et al. A pilot investigation of the short-term effects of an interdisciplinary intervention program on elderly patients with hip fracture in Taiwan. J Am Geriatr Soc 2005; 53:811-8.

18 Swanson CE, Day GA, Yelland CE, Broome JR, Massey L, Richardson HR, et al. The management of elderly patients with femoral fractures. A randomised controlled trial of early intervention versus standard care. Med J Aust 1998;169:515-8.

19 Young J, Green J, Forster A, Small N, Lowson K, Bogle S, et al. Postacute care for older people in community hospitals: a multicenter randomized, controlled trial. J Am Geriatr Soc 2007; 55:1995-2002.

20 Katz S, Downs TD, Cash HR, Grotz RC. Progress in development of the index of ADL. Gerontologist 1970;10:20-30.

21 Cohen HJ, Feussner JR, Weinberger M, Carnes M, Hamdy RC, Hsieh F, et al. A controlled trial of inpatient and outpatient geriatric evaluation and management. N Engl J Med 2002;346:905-12.

22 Karppi P. Effects of a geriatric inpatient unit on elderly home care patients: a controlled trial. Aging (Milano) 1995;7:207-11.

23 Kennie DC, Reid J, Richardson IR, Kiamari AA, Kelt C. Effectiveness of geriatric rehabilitative care after fractures of the proximal femur in elderly women: a randomised clinical trial. BMJ 1988;297:1083-6.

24 Reid J, Kennie DC. Geriatric rehabilitative care after fractures of the proximal femur: one year follow up of a randomised clinical trial. BM 1989;299:25-6.

25 Stenvall M, Olofsson B, Nyberg L, Lundstrom M, Gustafson Y. Improved performance in activities of daily living and mobility after a multidisciplinary postoperative rehabilitation in older people with femoral neck fracture: a randomized controlled trial with 1-year follow-up. J Rehabil Med 2007;39:232-8.

26 Vidan M, Serra JA, Moreno C, Riquelme G, Ortiz J. Efficacy of a comprehensive geriatric intervention in older patients hospitalized for hip fracture: a randomized, controlled trial. J Am Geriatr Soc 2005;53:1476-82.

27 White SJ, Powers JS, Knight JR, Harrell D, Varnell L, Vaughn C, et al. Effectiveness of an inpatient geriatric service in a university hospital. J Tenn Med Assoc 1994;87:425-8.

28 Applegate WB, Miller ST, Graney MJ, Elam JT, Burns R, Akins DE. A randomized, controlled trial of a geriatric assessment unit in a community rehabilitation hospital. N Engl J Med 1990;322:1572-8.

29 Rubenstein LZ, Josephson KR, Wieland GD, English PA, Sayre JA, Kane RL. Effectiveness of a geriatric evaluation unit. A randomized clinical trial. N Engl J Med 1984;311:1664-70.

30 Rubenstein LZ, Wieland GD, Josephson KR, Rosbrook B, Sayre J, Kane RL. Improved survival for frail elderly inpatients on a geriatric evaluation unit (GEU): who benefits? J Clin Epidemiol 1988;41:441-9.

31 Juni P, Altman DG, Egger M. Systematic reviews in health care: Assessing the quality of controlled clinical trials. BMJ 2001;323:42-6

32 Chinn S. A simple method for converting an odds ratio to effect size for use in meta-analysis. Stat Med 2000;19:3127-31.

33 Hasselblad V, Hedges LV. Meta-analysis of screening and diagnostic tests. Psychol Bull 1995;117:167-78.

34 Stuck AE, Egger M, Hammer A, Minder CE, Beck JC. Home visits to prevent nursing home admission and functional decline in elderly people: systematic review and meta-regression analysis. JAMA 2002;287:1022-8.

35 DerSimonian R, Laird N. Meta-analysis in clinical trials. Control Clin Trials 1986;7:177-88.

36 Higgins JP, Thompson SG. Quantifying heterogeneity in a metaanalysis. Stat Med 2002;21:1539-58

37 Begg CB, Mazumdar M. Operating characteristics of a rank correlation test for publication bias. Biometrics 1994;50:1088-101.

38 Egger M, Davey Smith G, Schneider M, Minder C. Bias in metaanalysis detected by a simple, graphical test. BMJ 1997;315:629-34.

39 Harbord RM, Egger M, Sterne JA. A modified test for small-study effects in meta-analyses of controlled trials with binary endpoints. Stat Med 2006;25:3443-57.

40 Juni P, Holenstein F, Sterne J, Bartlett C, Egger M. Direction and impact of language bias in meta-analyses of controlled trials: empirical study. Int J Epidemiol 2002;31:115-23.

41 Sterne JA, Egger M. Funnel plots for detecting bias in meta-analysis guidelines on choice of axis. J Clin Epidemiol 2001;54:1046-55.

42 Stettler C, Allemann S, Juni P, Cull CA, Holman RR, Egger M, et al. Glycemic control and macrovascular disease in types 1 and 2 diabetes mellitus: meta-analysis of randomized trials. Am Heart 2006;152:27-38.

43 Applegate WB, Graney MJ, Miller ST, Elam JT. Impact of a geriatric assessment unit on subsequent health care charges. Am J Public Health 1991;81:1302-6.

44 Fleming SA, Blake H, Gladman JR, Hart E, Lymbery M, Dewey ME, et al. A randomised controlled trial of a care home rehabilitation service to reduce long-term institutionalisation for elderly people. Age Ageing 2004;33:384-90.

45 Gilchrist WJ, Newman RJ, Hamblen DL, Williams BO. Prospective randomised study of an orthopaedic geriatric inpatient service. $B M$ 1988;297:1116-8.

46 Huusko TM, Karppi P, Avikainen V, Kautiainen H, Sulkava R. Randomised, clinically controlled trial of intensive geriatric rehabilitation in patients with hip fracture: subgroup analysis of patients with dementia. BMJ 2000;321:1107-11.

47 Huusko TM, Karppi P, Avikainen V, Kautiainen H, Sulkava R. Intensive geriatric rehabilitation of hip fracture patients: a randomized, controlled trial. Acta Orthop Scand 2002;73:425-31.

48 O'Reilly J, Lowson K, Green J, Young JB, Forster A. Post-acute care for older people in community hospitals-a cost-effectiveness analysis within a multi-centre randomised controlled trial. Age Ageing 2008;37:513-20.

49 Phibbs CS, Holty JE, Goldstein MK, Garber AM, Wang Y, Feussner JR, et al. The effect of geriatrics evaluation and management on nursing home use and health care costs: results from a randomized trial. Med Care 2006;44:91-5.

50 Ellis G, Langhorne P. Comprehensive geriatric assessment for older hospital patients. Br Med Bull 2004;71:45-59.

51 Stuck AE, Siu AL, Wieland GD, Adams J, Rubenstein LZ. Comprehensive geriatric assessment: a meta-analysis of controlled trials. Lancet 1993;342:1032-6.

52 Gill TM, Baker DI, Gottschalk M, Peduzzi PN, Allore H, Byers A. A program to prevent functional decline in physically frail, elderly persons who live at home. N Engl J Med 2002;347:1068-74.

53 Wells JL, Seabrook JA, Stolee P, Borrie MJ, Knoefel F. State of the art in geriatric rehabilitation. Part I: review of frailty and comprehensive geriatric assessment. Arch Phys Med Rehabil 2003;84:890-7.

54 Beswick AD, Rees K, Dieppe P, Ayis S, Gooberman-Hill R, Horwood J, et al. Complex interventions to improve physical function and maintain independent living in elderly people: a systematic review and meta-analysis. Lancet 2008;371:725-35.

Accepted: 29 January 2010 